\section{Fatores associados a excesso de peso e concentração de gordura abdominal em adultos na cidade de Salvador, Bahia, Brasil}

\author{
Factors associated with overweight and abdominal \\ fat in adults in Salvador, Bahia State, Brazil
}

\begin{abstract}
This cross-sectional study of 570 adults aimed to identify factors associated with overweight and abdominal fat in adults of both sexes in the city of Salvador, Bahia State, Brazil. Body mass index (BMI) and waist circumference were the dependent variables, and Poisson regression was used for statistical analysis. For women, high BMI and waist circumference were associated with increased age and current dieting ( $\leq 3$ meals a day), while family history of obesity was only associated with increased BMI. In men, BMI increased in the 30-39 and 50-59-year age brackets. Waist circumference increased in association with $\leq 3$ meals a day, arterial hypertension, and alcohol consumption, and was lower among blacks and mixed-race individuals. High BMI and waist circumference were both associated with family history of myocardial infarction. Thus, factors associated with overweight and abdominal fat differ between men and women, but adopting healthier lifestyles can help modify many of these factors.
\end{abstract}

Overweight; Abdominal Fat; Risk Factors; Adult
Lucivalda P. M. Oliveira ${ }^{1}$

Ana Marlúcia O. Assis 1

Maria da Conceição Monteiro da Silva ${ }^{1}$

Mônica Leila Portela de Santana 1

Nedja Silva dos Santos 1

Sandra M. C. Pinheiro 2

Mauricio Lima Barreto 3

Carine de Oliveira Souza 1

\section{Introdução}

A obesidade integra o conjunto das Doenças Crônicas não Transmissíveis (DCNT) cujo bloco abarca ainda diabetes mellitus, doenças cardiovasculares, hipertensão arterial, infarto do miocárdio e alguns tipos de cânceres 1 . Esse bloco de doenças constitui um dos principais problemas de saúde no mundo, pela alta ocorrência e pela expressão no padrão de morbidade adulta. Estimativas da Organização Mundial da Saúde (OMS) apontam as DCNT como responsáveis por $58,5 \%$ de todas as mortes e por $45,9 \%$ da carga global da morbidade adulta em todo o mundo 1 .

A ocorrência da obesidade e do sobrepeso vem aumentando no mundo inteiro 2,3, e também no Brasil. A análise comparativa entre estudos populacionais no Brasil, a exemplo do Estudo Nacional de Despesa Familiar (ENDEF) realizado em 1974/1975, da Pesquisa Nacional de Saúde e Nutrição (PNSN) conduzida em 1989 4 e da recente Pesquisa de Orçamentos Familiares (2002-2003) ${ }^{5}$ evidenciam a tendência de elevação do sobrepeso e da obesidade na população adulta brasileira. A distribuição segundo o sexo mostra que as mulheres concorrem com as mais altas prevalências de obesidade e que existe um gradiente segundo a escolaridade indicando maior ocorrência entre os indivíduos com baixo nível de escolaridade. A tendência de aumento da obesidade observada ao longo 
destes estudos é bem expressiva para ambos os sexos, notando-se, no entanto, estabilidade na prevalência da obesidade entre as mulheres de média e alta escolaridade 6 e aquelas com melhores condições econômicas ${ }^{4,5}$.

Os fatores de risco da obesidade e do sobrepeso têm sido creditados às mudanças sociais, culturais, ambientais e comportamentais, expressos especialmente nas mudanças no padrão alimentar e estilo de vida da população, que se caracterizam pela redução da atividade física, prática do tabagismo, consumo excessivo de bebidas alcoólicas e pelo aumento da taxa de urbanização 1. Entre os determinantes biológicos destacam-se a idade, em especial a faixa etária dos 30 aos 50 anos e o sexo 7,8.

Para as mulheres, destacam-se enquanto fatores contributivos a alta paridade, para aquelas em idade reprodutiva, a incorporação no mercado de trabalho e o menor esforço dispensado no trabalho doméstico, condição favorecida pela disponibilidade de eletrodomésticos modernos 9 .

A importância epidemiológica da obesidade é sinalizada não somente enquanto doença per $s i$, mas também porque o risco de diabetes, especialmente o tipo 2, de doenças cardiovasculares e de hipertensão aumenta diretamente com o grau do sobrepeso 1 .

A obesidade, enquanto doença resultante do acúmulo do excesso de gordura corpórea, tem sido tradicionalmente diagnosticada pelo indicador antropométrico índice de massa corporal (IMC), traduzido pela razão do peso pela altura ao quadrado 10 . Esse indicador antropométrico apresenta uma boa correlação com a gordura corporal e com as alterações metabólicas associadas à obesidade 11,12. Entretanto, a circunferência da cintura, que expressa a concentração de gordura abdominal, em especial a gordura visceral, é apontada como importante preditor de risco para as DCNT 7,10, particularmente para doenças cardiovasculares e diabetes 8,13.

Embora no Brasil existam informações populacionais confiáveis e atuais sobre a caracterização do estado antropométrico em adultos, elas se referem às macrorregiões do país e não enfocam os seus determinantes. E poucos centros urbanos brasileiros dispõem dessas informações oriundas de amostra representativa da população. Assim, o objetivo desta investigação foi identificar a prevalência e os fatores associados ao excesso de peso e da concentração de gordura abdominal em adultos na cidade de Salvador, Bahia, Brasil.

\section{Material e métodos}

\section{Tipo de estudo}

Este é um estudo transversal, realizado em 2001, com o objetivo de diagnosticar o estado de saúde e nutrição de adolescentes, adultos e idosos da cidade de Salvador. As informações aqui apresentadas restringem-se a uma amostra representativa da população adulta.

\section{Amostra}

Para o cálculo da amostra, adotou-se a prevalência de sobrepeso e obesidade em indivíduos de 19 a 59 anos da ordem de $30 \%$ estimada com base em dados da PNSN (1989), com nível de significância de $5 \%$ e erro de $4 \%$, obtendo-se uma amostra de 570 adultos. Adotou-se o desenho amostral por conglomerados, em três estágios. O subdistrito foi a unidade primária, o domicílio foi a secundária e os indivíduos, a unidade terciária. Desse modo, foram sorteados 11 subdistritos e dentre esses 44 setores, utilizando-se de mapas e informações fornecidas pela Prefeitura Municipal de Salvador. Em cada setor censitário selecionado foi sorteada uma rua como um marco inicial para identificação dos domicílios a serem visitados. Pelo primeiro domicílio, adotou-se o intervalo de cinco para a busca sistemática dos demais domicílios; considerando-se aqueles situados à direita e à esquerda de cada rua.

Foram visitados aproximadamente 200 domicílios em cada setor censitário, para identificar a amostra pré-definida, constituindo, em cada setor, uma média de 13 adultos que atendessem aos critérios de inclusão. Em caso de residir mais de um indivíduo adulto no mesmo domicílio, eles eram listados e um deles era sorteado para participar do estudo. Caso o morador não se encontrasse na residência no momento da entrevista era agendada uma nova visita, sendo permitidos até dois retornos. Se o encontro não se concretizasse, fazia-se a substituição por outro indivíduo da mesma faixa etária, sexo e da mesma zona de informação, de um domicilio não visitado. Após este estágio de captação da amostra, não era permitida a substituição. Foram excluídos do estudo: gestantes, nutrizes em amamentação até o quarto mês, portadores de deficiências física e mental, portadores de distúrbios endócrinos e indivíduos com algum processo mórbido em que foi detectada retenção hídrica localizada e/ou generalizada. 


\section{Coleta dos dados}

Os dados ambientais, do domicilio, sócio-econômicos e os relativos aos aspectos da saúde foram obtidos por entrevistas e registrados em questionários estruturados.

Os indivíduos foram classificados segundo a raça de acordo com a recomendação de Azevedo ${ }^{14}$, que leva em consideração as características físicas como tipo de cabelo e nariz, cor da pele e espessura dos lábios. Logo, os indivíduos foram classificados em branco, mulato claro, mulato médio, mulato escuro e negro. Os entrevistadores foram treinados e supervisionados por uma das autoras, anteriormente capacitada pela equipe de genética do Hospital Universitário Professor Edgar Santos.

Os dados antropométricos (peso, altura e circunferência da cintura) foram coletados por nutricionistas treinados. As medições foram realizadas em duplicata e uma terceira medida era aferida pelo supervisor quando os dois primeiros valores apresentavam diferenças maiores ou iguais a $0,5 \mathrm{~cm}$ para circunferência da cintura e estatura e $100 \mathrm{~g}$ para o peso 15 .

Para medida do peso foi utilizada balança digital, portátil, capacidade de $150 \mathrm{~kg}$ e intervalo de 100g (modelo E150 - INAN, marca Filizola, São Paulo, Brasil), com o indivíduo descalço e usando roupas padronizadas, descartáveis e especificamente confeccionadas para esse fim. A estatura foi aferida utilizando-se de estadiômetro portátil (Leicester Height Measure, Child Growth Foundation, Londres, Inglaterra), graduado em milímetros. A circunferência da cintura foi verificada por duas medidoras, utilizandose da fita métrica inelástica (TBW Importadora Ltda., São Paulo, Brasil) com 0,5cm de largura e $200 \mathrm{~cm}$ de comprimento posicionada horizontalmente na linha média entre a extremidade da última costela e a crista ilíaca superior, com os indivíduos em posição ortostática olhando para frente. A leitura da medida foi realizada no milímetro mais próximo, com o indivíduo respirando suavemente.

A padronização e a aferição das medidas antropométricas foram realizadas pela adoção das recomendações de Lohman et al. 15 .

\section{Definição das variáveis}

As variáveis respostas adotadas no estudo foram representadas pelo IMC e pela circunferência da cintura.

O IMC foi categorizado em $<25 \mathrm{~kg} / \mathrm{m}^{2}$ (sem risco) e $\geq 25 \mathrm{~kg} / \mathrm{m}^{2}$ (excesso de peso) como a categoria de risco, independentemente do sexo. Optou-se por juntar a magreza $\left(<18,5 \mathrm{~kg} / \mathrm{m}^{2}\right)$ com a eutrofia $\left(\geq 18,5 \mathrm{a}<25 \mathrm{~kg} / \mathrm{m}^{2}\right)$ e o sobrepeso $\left(\geq 25 \mathrm{a}<30 \mathrm{~kg} / \mathrm{m}^{2}\right)$ com a obesidade $(\geq 30 \mathrm{~kg} /$ $\left.\mathrm{m}^{2}\right)$, dado que a prevalência de magreza $(4,6 \%)$ e da obesidade $(12,8 \%)$, particularmente entre os homens, era baixa (Tabela 1) e não se adequavam às análises estatísticas definidas neste estudo.

A circunferência da cintura foi categorizada segundo o sexo. Para o feminino adotaram-se os pontos de corte de $\leq 80 \mathrm{~cm}$ (protetor) e $>80 \mathrm{~cm}$ (categoria de risco); para os homens a circunferência da cintura $\leq 94 \mathrm{~cm}$ foi considerada normal e a circunferência da cintura $>94 \mathrm{~cm}$ expressa o risco 10 .

As variáveis de exposição foram definidas, com base em revisão de literatura, entre aquelas que se mostraram mais consistentemente associadas ao excesso de peso e de gordura abdominal. Portanto, foram incluídas na análise exploratória as variáveis demográficas [sexo, idade (categorizadas em intervalos decilares); raça foi classificada em branca e mulato/negro constituíram uma única categoria; estado civil (solteiro - referência - casado)]; variáveis sócioeconômicas representadas pela escolaridade, renda familiar e ocupação; aquelas relacionadas com o estilo de vida: atividade dispensada no trabalho e no lazer, baseada na percepção do entrevistado sobre o grau do esforço físico despendido para desenvolver essas atividades e classificadas em muito intensa, intensa/moderada (referência) leve e não realiza atividade (identifica o risco) e, prática do tabagismo e do etilismo, com respostas dicotômicas (sim e não) a resposta afirmativa é indicativa do risco.

As variáveis dietéticas são resultantes do inquérito de freqüência alimentar e recordatório de 24 horas. Baseado na proposta de Fornés et al. 16, os dados da freqüência alimentar deram origem a um escore de freqüência de consumo alimentar correspondente a três grupos: o grupo das leguminosas, frutas e hortaliças; o grupo das carnes, embutidos, leite e derivados; e o grupo dos cereais e derivados. Considerou-se a freqüência de consumo referida para cada alimento utilizando ponderação, conforme consumo referido pelo entrevistado (diário, semanal, mensal ou não consumo), e equação sugerida pelo autor 16; assim, cada indivíduo apresentava um escore de consumo dos diferentes grupos de alimentos. Essas variáveis foram categorizadas em tercil. O método recordatório de 24 horas foi adotado para analisar adequação de carboidrato, gordura, proteína e disponibilidade de quilocaloria (Kcal) total da dieta com base na recomendação da OMS/Organização das Nações Unidas para Agricultura e Alimentação (FAO) ${ }^{1} \mathrm{e}$ das Dietary Reference Intakes (DRI) 17. 
Distribuição das características demográficas, sócio-econômicas, estilo de vida, história familiar, morbidades referidas e história alimentar segundo o sexo em adultos. Salvador, Bahia, Brasil, 2000-2001.

\begin{tabular}{|c|c|c|c|c|c|c|}
\hline \multirow[t]{2}{*}{ Variáveis } & \multicolumn{2}{|c|}{ Feminino } & \multicolumn{2}{|c|}{ Masculino } & \multicolumn{2}{|c|}{ Total } \\
\hline & $\mathrm{n}$ & $\%$ & $\mathrm{n}$ & $\%$ & $N(570)$ & $\%$ \\
\hline \multicolumn{7}{|l|}{ Índice de massa corporal (kg/m²) } \\
\hline Magreza ( $\leq 18)$ & 20 & 4,6 & 8 & 4,4 & 28 & 4,6 \\
\hline Eutrofia $(18,5-24,9)$ & 187 & 54,0 & 121 & 62,2 & 308 & 5,8 \\
\hline Sobrepeso $(25,0-29,9)$ & 94 & 26,3 & 54 & 25,0 & 148 & 25,8 \\
\hline Obesidade $(\geq 30)$ & 56 & 15,1 & 17 & 8,4 & 73 & 12,8 \\
\hline \multicolumn{7}{|l|}{ Circunferência da cintura * } \\
\hline Adequado & 225 & 64,3 & 173 & 87,1 & 398 & 71,9 \\
\hline Inadequado & 132 & 35,7 & 26 & 12,9 & 158 & 28,1 \\
\hline \multicolumn{7}{|l|}{ Idade (anos) } \\
\hline $20-29$ & 120 & 31,3 & 103 & 50,5 & 223 & 37,8 \\
\hline $30-39$ & 109 & 33,9 & 46 & 21,9 & 155 & 29,8 \\
\hline $40-49$ & 84 & 21,8 & 24 & 11,3 & 108 & 18,3 \\
\hline $50-59$ & 50 & 13,0 & 34 & 16,2 & 84 & 14,1 \\
\hline \multicolumn{7}{|l|}{ Raça } \\
\hline Brancos & 53 & 15,6 & 49 & 21,8 & 102 & 17,7 \\
\hline Mulatos e negros & 301 & 84,4 & 155 & 78,2 & 456 & 82,3 \\
\hline \multicolumn{7}{|l|}{ Estado civil } \\
\hline Casado & 192 & 56,7 & 81 & 40,5 & 273 & 51,2 \\
\hline Solteiro/Separado/Viúvo & 171 & 43,3 & 126 & 59,5 & 297 & 48,8 \\
\hline \multicolumn{7}{|l|}{ Ocupação } \\
\hline Empregado & 153 & 43,5 & 94 & 49,4 & 247 & 45,5 \\
\hline Aposentado/Afastado & 14 & 3,6 & 21 & 11,8 & 35 & 6,4 \\
\hline Desempregado & 195 & 52,9 & 92 & 38,8 & 287 & 48,1 \\
\hline \multicolumn{7}{|l|}{ Escolaridade } \\
\hline Analfabeto/Primário incompleto & 50 & 14,1 & 12 & 5,6 & 62 & 11,3 \\
\hline Primário completo/Ginásio incompleto & 69 & 19,5 & 35 & 16,0 & 104 & 18,3 \\
\hline Ginásio completo à colegial completo & 170 & 47,2 & 105 & 53,1 & 275 & 49,2 \\
\hline Superior completo/incompleto e pós-graduação & 74 & 19,2 & 55 & 25,3 & 129 & 21,2 \\
\hline \multicolumn{7}{|l|}{ Renda per capita (Reais) } \\
\hline$<100$ & 102 & 29,6 & 35 & 14,1 & 137 & 24,4 \\
\hline $100-215$ & 87 & 24,6 & 53 & 28,7 & 140 & 26,0 \\
\hline $215-500$ & 73 & 23,1 & 53 & 27,4 & 126 & 24,5 \\
\hline 500 ou mais & 90 & 22,7 & 63 & 29,8 & 153 & 25,1 \\
\hline \multicolumn{7}{|l|}{ Paridade (número de filhos) } \\
\hline Nenhum & 95 & 24,1 & - & - & - & - \\
\hline 1 ou 2 & 155 & 43,5 & - & - & - & - \\
\hline$\geq 3$ & 113 & 32,4 & - & - & - & - \\
\hline \multicolumn{7}{|l|}{ Tabagismo } \\
\hline Sim & 103 & 26,4 & 77 & 38,2 & 180 & 30,4 \\
\hline Não & 259 & 73,6 & 130 & 61,8 & 389 & 69,6 \\
\hline \multicolumn{7}{|l|}{ Uso de bebida alcoólica } \\
\hline Sim & 197 & 52,8 & 165 & 80,4 & 362 & 62,1 \\
\hline Não & 165 & 47,2 & 42 & 19,6 & 207 & 37,9 \\
\hline \multicolumn{7}{|l|}{ Atividade de esporte/lazer } \\
\hline Muito intensa/Intensa/Moderada & 124 & 34,5 & 119 & 58,2 & 243 & 42,6 \\
\hline Leve/Não realiza & 236 & 65,5 & 88 & 41,8 & 324 & 57,4 \\
\hline \multicolumn{7}{|l|}{ História familiar } \\
\hline Dislipidemia & 154 & 48,9 & 79 & 45,2 & 233 & 47,8 \\
\hline Angina & 85 & 24,0 & 52 & 25,3 & 137 & 24,4 \\
\hline
\end{tabular}

(continua) 


\begin{tabular}{|c|c|c|c|c|c|c|}
\hline \multirow[t]{2}{*}{ Variáveis } & \multicolumn{2}{|c|}{ Feminino } & \multicolumn{2}{|c|}{ Masculino } & \multicolumn{2}{|c|}{ Total } \\
\hline & $\mathrm{n}$ & $\%$ & $\mathrm{n}$ & $\%$ & $N(570)$ & $\%$ \\
\hline Obesidade & 118 & 34,6 & 63 & 29,4 & 181 & 32,9 \\
\hline Infarto & 105 & 29,7 & 71 & 36,5 & 176 & 32,0 \\
\hline \multicolumn{7}{|l|}{ Morbidades referidas } \\
\hline Dislipidemia & 59 & 19,4 & 28 & 19,6 & 87 & 19,5 \\
\hline Insuficiência cardíaca & 16 & 5,0 & 8 & 5,0 & 24 & 5,0 \\
\hline Hipertensão arterial & 72 & 22,2 & 28 & 16,3 & 100 & 20,2 \\
\hline \multicolumn{7}{|l|}{ Autoclassificação da saúde } \\
\hline Boa/Muito boa & 186 & 51,7 & 153 & 73,2 & 339 & 56,7 \\
\hline Regular/Mal/Muito mal & 176 & 48,3 & 54 & 26,8 & 230 & 43,3 \\
\hline \multicolumn{7}{|l|}{ Número de refeições-dia } \\
\hline$\geq 4$ & 193 & 52,8 & 116 & 57,7 & 309 & 54,4 \\
\hline$\leq 3$ & 170 & 47,2 & 91 & 42,3 & 261 & 45,6 \\
\hline \multicolumn{7}{|l|}{ Adoção de dieta } \\
\hline Sim & 128 & 37,6 & 49 & 27,3 & 177 & 34,1 \\
\hline Não & 235 & 62,4 & 158 & 72,7 & 393 & 65,9 \\
\hline \multicolumn{7}{|c|}{ Consumo de leguminosas, frutas e hortaliças ** } \\
\hline$\leq 3,53$ & 115 & 33,7 & 74 & 35,8 & 189 & 34,4 \\
\hline $3,54-5,53$ & 119 & 33,4 & 72 & 34,4 & 191 & 33,8 \\
\hline$\geq 5,54$ & 129 & 32,9 & 61 & 29,8 & 190 & 31,8 \\
\hline \multicolumn{7}{|c|}{ Consumo de carnes, embutidos, leite e derivados ** } \\
\hline$\leq 1,30$ & 137 & 40,5 & 65 & 31,8 & 202 & 37,6 \\
\hline $1,31-2,23$ & 103 & 28,6 & 64 & 30,3 & 167 & 29,1 \\
\hline$\geq 2,24$ & 123 & 30,9 & 78 & 37,9 & 201 & 33,3 \\
\hline \multicolumn{7}{|c|}{ Consumo de cereais e derivados ** } \\
\hline$\leq 1,43$ & 123 & 37,4 & 63 & 30,1 & 186 & 34,9 \\
\hline $1,44-2,70$ & 135 & 39,2 & 59 & 27,6 & 194 & 35,3 \\
\hline$\geq 2,71$ & 105 & 23,4 & 85 & 42,3 & 190 & 29,8 \\
\hline
\end{tabular}

* Inadequado: $>80 \mathrm{~cm}$ (mulheres) e $>94 \mathrm{~cm}$ (homens);

** Escore do padrão de consumo baseado na freqüência alimentar.

Investigou-se ainda número de refeições praticadas ao dia (categorizada em $\leq 3 \mathrm{e} \geq 4$ ), realização atual de dieta (sim e não), morbidades com diagnóstico médico e referidas pelo entrevistado (insuficiência cardíaca, diabetes, hipertensão arterial, dislipidemia, osteoporose, obstipação intestinal e anemia), história familiar de doenças referidas pelo entrevistado (derrame, infarto, angina, insuficiência cardíaca, diabetes, hipertensão arterial, dislipidemia - colesterol e/ou triglicérides elevados, obesidade, câncer e osteoporose), e auto-percepção do estado de saúde. Variáveis relacionadas à história reprodutiva da mulher (tipo de método contraceptivo em uso), paridade [nenhum filho (referência), 1 a $2 \mathrm{e} \geq 3$ filhos]. $\mathrm{O}$ tratamento dado às demais variáveis é apresentado de forma auto-explicativa nas tabelas dos resultados.

\section{Análise dos dados}

Utilizou-se a análise descritiva para caracterizar a distribuição da ocorrência dos eventos estudados. Para estimação das razões de prevalências, utilizou-se a análise de regressão log-Poisson, que é parte dos modelos lineares generalizados 18,19 . Inicialmente, realizou-se o modelo de regressão log-Poisson univariada para selecionar as variáveis que deveriam compor o modelo múltiplo. Por conseguinte, todas as variáveis que se mostraram associadas ao evento de interesse com significância estatística de até $20 \%$ foram incluídas no modelo múltiplo. Essa probabilidade foi estipulada para que possíveis fatores associados ao evento não fossem excluídos da análise 20 . Toda a análise foi realizada considerando o cálculo do peso realizado com base no desenho do estudo. Para aceitação das associações investigadas no modelo final, adotou-se o valor de $\mathrm{p}<0,05$. 
Considerando as idiossincrasias entre os sexos, particularmente pelas condições expressas na paridade 21 , e pelo fato de que a ocorrência das DCNT na idade adulta e o estado antropométrico se diferenciam segundo o sexo ${ }^{10}$, optou-se por processar os modelos estatísticos separadamente.

Utilizou-se o programa Epi Info (Centers for Disease Control and Prevention, Atlanta, Estados Unidos) para a entrada dos dados e o pacote estatístico Stata (Stata Corp., College Station, Estados Unidos) a fim de realizar as análises estatísticas. Para a avaliação antropométrica, adotaram-se os pontos de corte estabelecidos pela OMS 10 .

\section{Resultados}

A amostra em estudo, segundo o sexo, está caracterizada na Tabela 1. É composta por $66,2 \%$ das mulheres, com distribuição mais elevada do que a observada na população adulta da capital $(53,6 \%) 22$ com média de idade de 36,0 anos ( \pm $12,26)$. A média de idade entre os homens foi de $33,2$ anos ( $\pm 14,63)$ (dados não apresentados).

Identificou-se prevalência de $26,3 \%$ de sobrepeso nas mulheres e de $25 \%$ nos homens avaliado pelo IMC. Para a obesidade, a prevalência foi, respectivamente, de $15,1 \%$ e $8,4 \%$, entre mulheres e homens. Totalizando $41,4 \%$ de excesso de peso entre as mulheres e de $33,4 \%$ entre os homens. Adotando este mesmo indicador antropométrico, a magreza foi identificada, respectivamente, em $4,6 \%$ e $4,4 \%$ entre mulheres e homens entrevistados. Identificou-se ainda que $35,7 \%$ das mulheres e $12,9 \%$ dos homens tinham excesso de gordura abdominal, segundo o indicador circunferência da cintura.

Predominam em ambos os sexos indivíduos mulatos e negros; observando-se também maior freqüência de mulheres casadas (56,7\%) em comparação com os homens (40,5\%). Dos entrevistados 11,3\% apresentavam muito baixa escolaridade (1o ciclo do Ensino Fundamental incompleto) ou nenhuma escolaridade, e 18,3\% cursaram o 2 o ciclo do Ensino Fundamental incompleto; o nível superior completo/incompleto e a pós-graduação foram referidos por $21,2 \%$ dos entrevistados. No momento da entrevista, o desemprego atingia $48,1 \%$ dos adultos investigados, com predominância entre as mulheres $(52,9 \%)$; metade dos participantes $(50,4 \%)$ apresentava renda menor do que $\mathrm{R} \$ 215,00$ per capita (Tabela 1 ).

A maternidade foi experienciada por $75,9 \%$ das mulheres entrevistadas. Prevalências mais elevadas da prática do tabagismo $(38,2 \%)$ e do etilismo $(80,4 \%)$ foram identificadas entre os homens. No que se refere às condições relacionadas ao estilo de vida, a ausência de atividade física ou da realização de atividade de intensidade leve no esporte e lazer foi referida por $65,5 \%$ das mulheres e por $41,8 \%$ dos homens (Tabela 1 ).

Dentre as morbidades familiares referidas pelos homens, destacaram-se as dislipidemias $(45,2 \%)$ seguidas pelo infarto $(36,5 \%)$ e pela obesidade $(29,4 \%)$ e, para as mulheres, sobressaíram também as dislipidemias $(48,9 \%)$, a obesidade $(34,6 \%)$ e o infarto $(29,7 \%)$. Os problemas de saúde de maior ocorrência referidos pelos homens e pelas mulheres foram as dislipidemias $19,6 \%$ e $19,4 \%$ e, a hipertensão arterial $16,3 \%$ e $22,2 \%$, respectivamente (Tabela 1 ).

Identificou-se ainda que $37,6 \%$ das mulheres e $27,3 \%$ dos homens já tinham adotado algum tipo de dieta; $47,2 \%$ das mulheres e $42,3 \%$ dos homens realizavam três ou menos refeições ao dia. A distribuição das variáveis relativas ao consumo dietético está apresentada em tercil também na Tabela 1. As demais variáveis investigadas neste estudo, que apresentaram valores de significância maiores do que $20 \%$ não foram apresentadas.

Os resultados da análise univariada, realizada segundo o gênero, indicaram para as mulheres investigadas que as variáveis demográficas (idade e paridade), condição ocupacional (aposentada/afastada do trabalho), estilo de vida (tabagismo e uso de bebida alcoólica), história familiar de doenças (obesidade, dislipidemia e angina), realização de três ou menos refeições ao dia, adoção atual de dieta e morbidade referida com base no diagnóstico médico (insuficiência cardíaca, hipertensão e dislipidemias), elevavam a prevalência tanto do excesso de peso (IMC) quanto do excesso de gordura abdominal (circunferência da cintura) (Tabela 2).

A análise univariada processada para os homens indicou que as prevalências tanto do excesso de peso quanto da gordura abdominal eram mais elevadas nas idades de 30 a 39 e de 50 a 59 anos, quando comparada com a faixa etária de 20 a 29 anos. Dentre as morbidades referidas, com diagnóstico médico, a hipertensão arterial se associou positivamente com os dois indicadores avaliados. A realização de três ou menos refeições ao dia elevou a prevalência do excesso de peso e da concentração de gordura abdominal, quando comparada com a prevalência destes eventos entre aqueles que realizavam mais de três refeições ao dia. Para os homens investigados, a prevalência de história familiar de infarto apresentou associação significante com excesso de peso e gordura abdominal (Tabela 2).

No modelo final os resultados da análise de Poisson, processada para as mulheres (Tabela 3), indicaram que à medida que aumentava a idade 
Tabela 2

Razão de prevalência bruta para o excesso de peso e excesso de gordura abdominal em adultos. Salvador, Bahia, Brasil, $2000-2001$.

\begin{tabular}{|c|c|c|c|c|}
\hline \multirow[t]{3}{*}{ Variáveis } & \multicolumn{4}{|c|}{ RP bruto (IC95\%) } \\
\hline & \multicolumn{2}{|c|}{ Masculino } & \multicolumn{2}{|c|}{ Feminino } \\
\hline & IMC & Circunferência da cintura & IMC & Circunferência da cintura \\
\hline \multicolumn{5}{|l|}{ Idade (anos) } \\
\hline $20-29$ & - & - & - & - \\
\hline $30-39$ & $3,10(1,78-5,37)$ & $4,13(1,32-12,91)$ & $1,71(1,04-2,81)$ & $1,72(0,98-3,02)$ \\
\hline $40-49$ & $1,52(0,63-3,66)$ & $2,30(0,53-9,97)$ & $2,34(1,45-3,77)$ & $2,39(1,38-4,12)$ \\
\hline $50-59$ & $3,22(1,83-5,65)$ & $3,39(1,32-14,66)$ & $2,84(1,76-4,58)$ & $3,36(1,98-5,71)$ \\
\hline \multicolumn{5}{|l|}{ Raça } \\
\hline Brancos & - & - & - & - \\
\hline Mulatos e negros & $0,86(0,53-1,41)$ & $0,49(0,22-1,09)$ & $0,76(0,54-1,11)$ & $0,65(0,45-0,94)$ \\
\hline \multicolumn{5}{|l|}{ Estado civil } \\
\hline Solteiro & - & - & - & - \\
\hline Casado & $1,40(0,90-2,18)$ & $1,94(0,84-4,48)$ & $1,05(0,78-1,42)$ & $1,35(0,96-1,91)$ \\
\hline \multicolumn{5}{|l|}{ Ocupação } \\
\hline Empregado & & & - & - \\
\hline Aposentado/Afastado & $1,52(0,88-2,65)$ & $0,19(0,39-3,59)$ & $1,83(1,20-2,78)$ & $2,31(1,48-3,60)$ \\
\hline Desempregado & $0,72(0,44-1,19)$ & $0,43(0,17-1,09)$ & $1,03(0,75-1,41)$ & $1,18(0,83-1,68)$ \\
\hline \multicolumn{5}{|l|}{ Paridade (número de filhos) } \\
\hline Nenhum & - & - & - & - \\
\hline 1 ou 2 & - & - & $1,34(0,86-2,10)$ & $1,90(1,12-3,21)$ \\
\hline$\geq 3$ & - & - & $1,70(1,09-2,65)$ & $2,22(1,31-3,76)$ \\
\hline \multicolumn{5}{|l|}{ Autoclassificação da saúde } \\
\hline Boa/Muito boa & - & - & - & - \\
\hline Regular/Muito mal & $0,84(0,51-1,42)$ & $1,02(0,42-2,45)$ & $1,22(0,90-1,65)$ & $1,52(1,09-2,13)$ \\
\hline \multicolumn{5}{|l|}{ Intensidade da atividade esporte/lazer } \\
\hline Muito intensa/Intensa/Moderada & - & - & - & - \\
\hline Leve/Não realiza & $0,88(0,56-1,38)$ & $0,99(0,44-2,24)$ & $1,11(0,81-1,53)$ & $1,43(0,98-2,09)$ \\
\hline \multicolumn{5}{|l|}{ Tabagismo } \\
\hline Não & - & - & - & - \\
\hline $\operatorname{Sim}$ & $1,07(0,68-1,68)$ & $1,86(0,48-2,46)$ & $1,54(1,16-2,03)$ & $1,72(1,26-2,35)$ \\
\hline \multicolumn{5}{|l|}{ Uso de bebida alcoólica } \\
\hline Não & - & - & - & - \\
\hline Sim & $1,07(0,61-1,86)$ & $2,69(0,75-9,61)$ & $1,28(0,94-1,75)$ & $1,45(1,03-2,05)$ \\
\hline \multicolumn{5}{|l|}{ História familiar (sim) } \\
\hline Obesidade & $1,02(0,63-1,65)$ & $1,97(0,89-4,36)$ & $1,51(1,13-2,02)$ & $1,46(1,06-2,02)$ \\
\hline Dislipidemia & $1,14(0,72-1,80)$ & $1,13(0,44-2,90)$ & $1,41(1,04-1,92)$ & $1,44(1,02-2,04)$ \\
\hline Angina & $1,01(0,62-1,64)$ & $1,25(0,51-3,06)$ & $1,44(1,07-1,94)$ & $1,40(0,99-1,99)$ \\
\hline Infarto & $1,57(1,03-2,41)$ & $3,36(1,46-7,73)$ & $0,96(0,69-1,35)$ & $0,88(0,59-1,30)$ \\
\hline \multicolumn{5}{|l|}{ Número de refeições-dia } \\
\hline$\leq 3$ & $2,04(1,30-3,20)$ & $3,49(1,36-8,98)$ & $1,61(1,19-2,19)$ & $1,67(1,20-2,33)$ \\
\hline \multicolumn{5}{|l|}{ Adoção de dieta } \\
\hline Sim & $1,53(0,97-2,40)$ & $2,03(0,91-4,54)$ & $1,90(1,43-2,53)$ & $2,00(1,46-2,75)$ \\
\hline \multicolumn{5}{|l|}{ Escolaridade } \\
\hline Analfabeto/Primário incompleto & $0,90(0,34-2,41)$ & $092(0,19-4,54)$ & $0,90(0,54-1,50)$ & $0,93(0,54-1,58)$ \\
\hline Primário completo/Ginásio incompleto & $1,08(0,59-1,95)$ & $0,92(0,27-3,05)$ & $1,01(0,64-1,60)$ & $0,88(0,53-1,47)$ \\
\hline Ginásio completo à colegial completo & $0,81(0,48-1,35)$ & $0,79(0,31-2,01)$ & $0,94(0,64-1,39)$ & $0,85(0,56-1,30)$ \\
\hline \multicolumn{5}{|l|}{ Renda per capita (Reais) } \\
\hline$<100$ & $1,16(0,65-2,08)$ & $0,40(0,11-1,50)$ & $0,81(0,54-1,21)$ & $0,69(0,45-1,08)$ \\
\hline $100-215$ & $0,61(0,32-1,16)$ & $0,60(0,21-1,70)$ & $0,79(0,51-1,23)$ & $0,61(0,36-1,03)$ \\
\hline $215-500$ & $1,12(0,64-1,94)$ & $1,05(0,40-2,76)$ & $1,01(0,67-1,53)$ & $0,91(0,58-1,43)$ \\
\hline 500 ou mais & - & - & - & - \\
\hline
\end{tabular}

(continua) 


\begin{tabular}{|c|c|c|c|c|}
\hline \multirow[t]{3}{*}{ Variáveis } & \multicolumn{4}{|c|}{ RP bruto (IC95\%) } \\
\hline & \multicolumn{2}{|c|}{ Masculino } & \multicolumn{2}{|c|}{ Feminino } \\
\hline & IMC & Circunferência da cintura & IMC & Circunferência da cintura \\
\hline \multicolumn{5}{|l|}{ Morbidades referidas } \\
\hline Dislipidemia & $1,56(0,93-2,62)$ & $1,57(0,54-4,57)$ & $1,44(1,03-2,04)$ & $1,68(1,17-2,41)$ \\
\hline Hipertensão & $1,94(1,20-3,12)$ & $3,48(1,52-7,93)$ & $1,33(0,96-1,83)$ & $1,68(1,20-2,34)$ \\
\hline Insuficiência cardíaca & $1,05(0,36-3,05)$ & $1,51(0,25-9,26)$ & $1,90(1,33-2,71)$ & $2,02(1,35-3,01)$ \\
\hline \multicolumn{5}{|c|}{ Consumo de leguminosas, frutas e hortaliças * } \\
\hline$\leq 3,53$ & $1,21(0,70-2,10)$ & $0,98(0,38-2,53)$ & $1,06(0,73-1,54)$ & $1,10(0,71-1,71)$ \\
\hline $3,54-5,53$ & $1,12(0,63-1,99)$ & $1,07(0,38-2,97)$ & $1,14(0,80-1,63)$ & $1,46(0,99-2,16)$ \\
\hline$\geq 5,54$ & - & - & - & - \\
\hline \multicolumn{5}{|c|}{ Consumo de carnes, embutidos, leite e derivados * } \\
\hline$\leq 1,30$ & - & - & - & - \\
\hline $1,31-2,23$ & $1,07(0,62-1,85)$ & $1,12(0,41-3,09)$ & $0,96(0,69-1,35)$ & $1,00(0,69-1,44)$ \\
\hline$\geq 2,24$ & $1,19(0,70-2,02)$ & $1,33(0,50-3,53)$ & $0,86(0,59-1,26)$ & $0,80(0,52-1,23)$ \\
\hline
\end{tabular}

IMC: índice de massa corporal.

IMC $\geq 25 \mathrm{~kg} / \mathrm{m}^{2}$; circunferência da cintura $>80 \mathrm{~cm}$ (mulheres) e $>94 \mathrm{~cm}$ (homens).

* Escore do padrão de consumo baseado na freqüência alimentar.

elevava também a prevalência do excesso de peso e da concentração da gordura abdominal. Mulheres que adotavam algum tipo de dieta apresentavam prevalência mais elevada de excesso de peso (RP: 1,64; IC\%: 1,23-2,19) e gordura abdominal (RP: 1,80; IC\%: 1,32-2,47) do que aquelas que não praticavam nenhum tipo de dieta. A história familiar de obesidade elevou significantemente a prevalência do excesso de peso (RP: 1,42; IC\%: $1,08-1,86)$, quando comparada com a prevalência daquelas sem registro desses eventos entre os familiares (Tabela 3).

Para os adultos do sexo masculino, as faixas etárias de 30 a 39 anos e de 50 a 59 anos elevaram, respectivamente, em 183\% (RP: 2,83; IC\%: 1,65-4,84) e $196 \%$ (RP: 2,96; IC\%: 1,71-5,15) a prevalência do excesso de peso. A realização de três ou menos refeições/dia associou-se significantemente ao excesso de gordura abdominal (RP: 3,53; IC\%: 1,33-9,34). A história familiar de infarto associou-se à prevalência do excesso de peso (RP: 1,56; IC\%: 1,06-2,31) e ao excesso de gordura abdominal (RP: 3,19; IC\%: 1,39-7,35). A análise evidenciou também menor ocorrência de excesso de gordura abdominal (RP: 0,41; IC\%: 0,18-0,95) em indivíduos mulatos e negros quando comparados aos brancos (Tabela 4).

\section{Discussão}

Os resultados desta investigação mostram alta prevalência de sobrepeso $(25,8 \%)$, obesidade
$(12,8 \%)$ e excesso de gordura abdominal $(28,1 \%)$ entre a população adulta de Salvador. E evidenciam também o diferencial dos distúrbios antropométricos segundo o sexo.

Neste sentido, a prevalência da obesidade identificada entre mulheres $(15,1 \%)$ e homens (8,4\%) da capital do Estado da Bahia em 2001 é mais elevada do que a verificada pela POF 2002$2003{ }^{5}$ para as mulheres $(11,7 \%)$ e homens $(6,7 \%)$ do Nordeste brasileiro. Para as mulheres de Salvador a ocorrência da obesidade não apresenta diferença substancial com a encontrada entre as mulheres da Região Sul (15,1\%). No entanto, a prevalência da obesidade entre os homens desta região é mais elevada $(10,1 \%)$ se comparada com aquela dos homens de Salvador.

Nota-se ainda que, em 2002-2003, período próximo ao que foi realizado o estudo de Salvador (2000-2001), a ocorrência do excesso de peso (IMC $\geq 25 \mathrm{~kg} / \mathrm{m}^{2}$ ) foi menor entre as mulheres $(38,8 \%)$ e homens $(32,9 \%)$ da Região Nordeste, quando comparada com as observadas entre as mulheres $(41,4 \%)$ e homens $(33,4 \%)$ de Salvador. Indicando, portanto, maior prevalência de sobrepeso e obesidade entre a população adulta de Salvador comparada aos dados da POF para a Região Nordeste 5 .

Os resultados deste estudo indicaram que o excesso de gordura abdominal, avaliado pela circunferência da cintura, acompanha a tendência da obesidade, com prevalência 2,7 vezes mais elevada entre as mulheres $(35,7 \%)$ quando comparada à estimada entre os homens (12,9\%). 
Tabela 3

Fatores associados ao índice de massa corporal (IMC) e à circunferência da cintura, de acordo com regressão de Poisson, em mulheres. Salvador, Bahia, Brasil, 2000-2001.

\begin{tabular}{|c|c|c|c|c|}
\hline \multirow[t]{2}{*}{ Variáveis } & \multicolumn{4}{|c|}{ RP ajustado } \\
\hline & IMC & IC95\% & Circunferência da cintura & IC95\% \\
\hline \multicolumn{5}{|c|}{ Idade (anos) } \\
\hline 20-29 & - & - & - & - \\
\hline $30-39$ & 1,64 & $1,03-2,62$ & 1,67 & $0,97-2,90$ \\
\hline $40-49$ & 2,16 & $1,38-3,39$ & 2,18 & $1,29-3,70$ \\
\hline $50-59$ & 2,56 & $1,62-4,06$ & 3,00 & $1,77-5,09$ \\
\hline \multicolumn{5}{|c|}{ Adoção de dieta } \\
\hline Sim & 1,64 & $1,23-2,19$ & 1,80 & $1,32-2,47$ \\
\hline Não & - & - & - & - \\
\hline \multicolumn{5}{|c|}{ História familiar de obesidade } \\
\hline Sim & 1,42 & $1,08-1,86$ & - & - \\
\hline Não & - & - & - & - \\
\hline
\end{tabular}

IMC $\geq 25 \mathrm{~kg} / \mathrm{m}^{2}$ e circunferência da cintura > 94cm; RP: razão de prevalência ajustado pelas demais variáveis apresentadas.

Tabela 4

Fatores associados ao índice de massa corporal (IMC) e à circunferência da cintura, de acordo com regressão de Poisson, em homens. Salvador, Bahia, Brasil, 2000-2001.

\begin{tabular}{|c|c|c|c|c|}
\hline \multirow[t]{2}{*}{ Variáveis } & \multicolumn{4}{|c|}{ RP ajustado } \\
\hline & IMC & IC95\% & Circunferência da cintura & IC95\% \\
\hline \multicolumn{5}{|l|}{ Idade (anos) } \\
\hline $20-29$ & - & - & - & - \\
\hline $30-39$ & 2,83 & $1,65-4,84$ & - & - \\
\hline $40-49$ & 1,53 & $0,65-3,57$ & - & - \\
\hline $50-59$ & 2,96 & $1,71-5,15$ & - & - \\
\hline \multicolumn{5}{|c|}{ Número de refeições-dia } \\
\hline$\geq 4$ & - & - & - & - \\
\hline$\leq 3$ & & & 3,53 & $1,33-9,34$ \\
\hline \multicolumn{5}{|l|}{ Hipertensão } \\
\hline $\operatorname{Sim}$ & & & 3,43 & $1,67-7,05$ \\
\hline Não & - & & - & - \\
\hline \multicolumn{5}{|c|}{ História familiar de infarto } \\
\hline $\operatorname{Sim}$ & 1,56 & $1,06-2,31$ & 3,19 & $1,39-7,35$ \\
\hline Não & - & - & - & - \\
\hline \multicolumn{5}{|l|}{ Uso de álcool } \\
\hline Sim & - & - & 3,46 & $1,02-11,77$ \\
\hline Não & - & - & - & - \\
\hline \multicolumn{5}{|l|}{ Raça } \\
\hline Branco & - & - & - & - \\
\hline Mulatos e negros & - & - & 0,41 & $0,18-0,95$ \\
\hline
\end{tabular}


Assim, é importante ressaltar que, independentemente do sexo, a simultaneidade da exposição ao excesso de peso e excesso de gordura abdominal (26,1\%, dado não apresentado), identificada na população investigada a coloca em condição de maior predisposição para as DCNT.

A evolução do perfil antropométrico construído por intermédio das informações dos três inquéritos de abrangência nacional (ENDEF 1974/1975; PNSN 1989; Pesquisa sobre Padrões de Vida - PPV - 1996/1997; POF 2002/2003) 4,5 indica aumento da prevalência da obesidade entre os homens em todas as grandes regiões do país. Entre as mulheres esse aumento foi observado somente no Nordeste; enquanto a tendência de estabilidade foi registrada entre as mulheres do Norte e Sudeste e declínio entre aquelas das regiões Sul e Centro-Oeste.

Para o excesso de peso (IMC $\geq 25 \mathrm{~kg} / \mathrm{m}^{2}$ ), o perfil construído é caracterizado pelo aumento da prevalência para os homens em todas as regiões do país enquanto para as mulheres o aumento deste evento só foi identificado na Região Nordeste, sobressaindo o declínio da ocorrência para as mulheres residentes nas demais regiões do país 5 .

Vários fatores podem estar contribuindo para a construção do perfil antropométrico da população adulta da cidade de Salvador. Os resultados deste estudo, segundo o sexo, indicam que a prevalência do excesso de peso e da gordura abdominal se torna crescente com o aumento da idade da mulher, concordando com os achados para obesidade abdominal na Região Sul do Brasil 23. O aumento da prevalência do excesso de peso para os homens não apresenta tendência crescente à proporção que aumenta a idade, declinando e perdendo a significância estatística entre os homens de 40 a 49 anos, elevando-se para a faixa de 50 a 59 anos. Resultados similares foram também registrados pela POF 2002-2003 5 para o declínio da prevalência do excesso de peso entre os homens a partir dos 55 anos de idade. Ainda assim, não passa despercebido que esses resultados possam estar indicando alguma flutuação na amostra, uma vez que tanto a ocorrência das DCNT quanto da mortalidade aumenta à medida que aumenta a idade para homens e mulheres 2,9,12,24.

A redução do número de refeições ingeridas ao dia foi outro fator que se mostrou inicialmente associado tanto ao excesso de peso quanto ao de gordura abdominal, para mulheres e homens investigados, e no modelo final mantém significância apenas para gordura abdominal entre os homens. Resultados similares foram encontrados para o excesso de peso na população masculina de Goiânia 25. Essa associação poderia ser interpretada como resposta fisiológica à adoção de uma prática alimentar inadequada, uma vez que a redução do número de refeição ao dia conduz o indivíduo a fazer alta ingestão de alimentos e conseqüentemente com elevada concentração calórica por refeição. Ainda com relação ao padrão alimentar, destaca-se a associação observada entre fazer dieta para perda ponderal e o excesso de peso e de gordura abdominal entre as mulheres. Esta condição pode ser interpretada à luz da causalidade reversa dado que, uma vez diagnosticado o excesso de peso corporal ou da gordura abdominal, o indivíduo pode ter iniciado medidas de restrição alimentar para o controle destes eventos. Tais características não são passíveis de serem avaliadas em estudos que adotam desenho de corte transversal.

O uso de bebida alcoólica mostra-se associado com o aumento da concentração de gordura abdominal somente para os homens e a história familiar de obesidade com o aumento de peso somente entre as mulheres, ainda que esses dois eventos sejam indicados como fatores de risco para a alteração do padrão antropométrico tanto de homens quanto de mulheres em alguns estudos $23,26,27$. No que se refere ao consumo de bebida alcoólica, a explicação para a diferença entre os sexos é possível que esteja relacionada não só à freqüência do consumo mais elevado entre os homens, como também à quantidade de bebida consumida 28 .

Neste estudo, a história familiar da doença estende-se não somente aos pais, mas também os irmãos, avós e tios de 1o grau dos entrevistados. Assim, essa associação pode indicar a forte influência genética na ocorrência do evento e/ou a influência de fatores ligados ao estilo de vida na unidade familiar, relação amplamente documentada na literatura 29,30,31.

A história familiar de infarto agudo do miocárdio associou-se tanto com o excesso de peso quanto com o de gordura abdominal, especificamente, para os homens. Essa associação é também relatada por Martins \& Marinho 12 em população investigada no Município de São Paulo e por Gigante et al. ${ }^{26}$ em Pelotas. Dados do Ministério da Saúde revelam que, entre as DCNT, as cardiovasculares constituem uma das principais causas de morte no Brasil, entretanto o coeficiente de mortalidade é maior entre os homens ${ }^{32}$. Neste estudo, a associação observada entre hipertensão e o excesso de gordura abdominal para o sexo masculino confirma uma relação já evidenciada 26 .

Vale a pena comentar neste estudo, a associação encontrada entre raça e excesso de gordura abdominal entre os homens. Observou-se que ser mulato ou negro é um fator de proteção para 
o acúmulo de gordura abdominal, discordando de dados da literatura ${ }^{9}$. Este resultado é possível ser explicado pelas diferentes metodologias adotadas como a identificação de cor da pele pelo entrevistador, ou auto-referida, e ainda por características genéticas baseadas no fenótipo como foi o caso deste estudo, assim como pela ausência de confundimento nas variáveis proxy de condições econômicas.

De uma maneira geral, pode-se observar que dentre os fatores selecionados neste estudo, para explicar a ocorrência do excesso de peso e concentração de gordura abdominal identificou-se que alguns deles são dependentes do sexo indicando, portanto, uma possível dependência de gênero.

Independentemente da influência genética que se expressa na história familiar, entende-se que a obesidade resulta de uma rede complexa de fatores ligados ao campo genético, ao estilo de

\section{Resumo}

O objetivo foi identificar os fatores associados ao excesso de peso e concentração de gordura abdominal em adultos de ambos os sexos. Um estudo transversal envolvendo amostra representativa de 570 adultos de Salvador, Bahia, Brasil. A regressão de Poisson foi utilizada na análise, adotando-se o índice de massa corporal (IMC) e a circunferência da cintura como variáveis dependentes. Para as mulheres, a inadequação do IMC e da circunferência da cintura ocorreu com o aumento da idade e entre as que faziam dieta no momento do estudo, a história familiar de obesidade associouse apenas com o IMC elevado. Para os homens, o IMC elevou-se na faixa etária de 30 a 39 e 50 a 59 anos. A circunferência da cintura elevou-se com a realização de três ou menos refeições-dia, com a hipertensão arterial, uso de bebida alcoólica e diminuiu entre mulatos e negros. Tanto IMC quanto circunferência da cintura elevaram-se com a historia familiar de infarto. Portanto são distintos os fatores que se associam ao excesso de peso e da gordura abdominal em homens e mulheres. Mas muitos deles podem ser modificados com a adoção do estilo de vida saudável.

Sobrepeso; Gordura Abdominal; Fatores de Risco; Adulto vida e ao ambiente que se associam e interagem. Vale ressaltar que, embora a influência genética seja reconhecida como fator de risco para a obesidade, particularmente a obesidade abdominal, os fatores do meio ambiente e do estilo de vida são os mais expressivos fatores de risco para a ocorrência do sobrepeso e da obesidade em todo o mundo ${ }^{1}$. Neste sentido, as medidas de controle e prevenção dos riscos modificáveis para a população estudada estão situadas na esfera da adoção de estilo de vida e da alimentação saudável, ainda que não tenha sido encontrada associação entre estas variáveis incluídas no modelo e o excesso de peso e/ou gordura abdominal. Estas são reconhecidas como estratégias capazes de reduzir a expressiva parcela das mortes prematuras e o impacto negativo na qualidade de vida dos indivíduos ${ }^{33}$, atributos que podem também ser destacados para o sobrepeso enquanto evento que precede a obesidade.

\section{Colaboradores}

S. M. C. Pinheiro participou da elaboração e interpretação da análise estatística. A. M. O. Assis, M. C. M. Silva, M. L. P. Santana, N. S. Santos coordenaram trabalho de campo, participaram da elaboração de instrumento para coleta de dado e realizaram coleta de dados. C. O. Souza participou da coleta de dados. L. P. M. Oliveira, A. M. O. Assis e M. L. Barreto realizaram implementação e interpretação dos resultados e revisão final do artigo, os demais autores também participaram da elaboração e revisão do manuscrito. 


\section{Referências}

1. World Health Organization/Food and Agriculture Organization. Diet, nutrition and the prevention of chronic diseases. Geneva: World Health Organization; 2003. (Technical Report Series, 916).

2. Jee SH, Sull JW, Park J, Lee SY, Ohrr H, Guallar E, et al. Body-mass index and mortality in korean men and women. N Engl J Med 2006; 355:779-87.

3. Adams KF, Schatzkin A, Harris TB, Kipnis V, Mouw T, Ballard-Barbash HA, et al. Overweight, obesity, and mortality in a large prospective cohort of persons 50 to 71 years old. N Engl J Med 2006; 355:763-78.

4. Monteiro CA, Conde WL, Castro IRR. A tendência cambiante da relação entre escolaridade e risco de obesidade no Brasil (1975-1997). Cad Saúde Pública 2003; 19 Suppl 1:S67-75.

5. Instituto Brasileiro de Geografia e Estatística. Pesquisa de orçamento familiares 2002-2003. Análise da disponibilidade domiciliar de alimentos e do estado nutricional no Brasil. Rio de Janeiro: Instituto Brasileiro de Geografia e Estatística; 2006.

6. Bossan FM, Anjos LA, Vasconcellos MTL, Wahrlich $\mathrm{V}$. Nutritional status of the adult population in $\mathrm{Ni}$ terói, Rio de Janeiro, Brazil: the Nutrition, Physical Activity, and Health Survey. Cad Saúde Pública 2007; 23:1867-76.

7. Janssen I, Heymsfield SB, Allison DB, Kotler DP, Ross R. Body mass index and waist circumference independently contribute to the prediction of nonabdominal, abdominal subcutaneous and visceral fat. Am J Clin Nutr 2002; 75:683-8.

8. Lerario DDG, Gimeno SG, Franco LJ, Iunes M, Ferreira SRG. Excesso de peso e gordura abdominal para a síndrome metabólica em nipo-brasileiros. Rev Saúde Pública 2002; 36:4-11.

9. Castanheira M, Olinto MTA, Gigante DP. Associação de variáveis sócio-demográficas e comportamentais com a gordura abdominal em adultos: estudo de base populacional no Sul do Brasil. Cad Saúde Pública 2003; 19 Suppl 1:S55-65.

10. World Health Organization. Obesity: preventing and managing the global epidemic. Report of a WHO consultation on obesity. Geneva: World Health Organization; 1998.

11. Cabrera MAS, Jacob Filho W. Obesidade em idosos: prevalência, distribuição e asociação com hábitos e co-morbidades. Arq Bras Endocrinol Metab 2001; 45:494-501.

12. Martins IS, Marinho SP. O potencial diagnóstico dos indicadores da obesidade centralizada. Rev Saúde Pública 2003; 37:760-7.

13. Souza LJ, Gicovante Neto C, Chalita FEB, Reis AFF, Bastos DA, Souto Filho JTD, et al. Prevalência de obesidade e fatores de risco cardiovasculares em Campos, Rio de Janeiro. Arq Bras Endocrinol Metab 2003; 47:669-76.

14. Azevedo ES. Subgroups studies of black admixture within a mixed population of Bahia, Brazil. Ann Hum Genet 1980; 44:55-60.

15. Lohman TG, Roche AF, Martorell R. Anthropometric standardization reference manual. Champaign: Human Kinetics; 1988.
16. Fornés NS, Martins IS, Velásquez-Meléndez G, Latorre MRDO. Escores de consumo alimentar e níveis lipêmicos em população de São Paulo, Brasil. Rev Saúde Pública 2002; 36:12-8.

17. Food and Nutrition Board, Institute of Medicine of the National Academies. Dietary reference intakes, for energy, carbohydrate, fiber, fat, fatly acids, cholesterol, protein and aminoacids. Washington DC: National Academies Press; 2002.

18. Dobson AJ. An introduction to generalized linear models. London: Chapman \& Hall; 1990.

19. Nelder JA, Wedderburn RWM. Generalized linear models. J R Stat Soc [Ser A] 1972; 135:370-84.

20. Hosmer DW, Lemeshow S. Applied logistic regression. New York: John Wiley \& Sons; 1989.

21. Kac G, Velásquez-Meléndez G, Coelho MASC. Fatores associados à obesidade abdominal em mulheres em idade reprodutiva. Rev Saúde Pública $2001 ; 35: 46-51$.

22. Instituto Brasileiro de Geografia e Estatística. Censo demográfico 1991: resultados do universo relativos às características da população e dos domicílios. v. 17, Bahia. Rio de Janeiro: Instituto Brasileiro de Geografia e Estatística; 1994.

23. Olinto MTA, Costa JSD, Kac G, Pattussi MP. Epidemiologia da obesidade abdominal em mulheres adultas residentes no sul do Brasil. Arch Latinoam Nutr 2007; 57:349-56.

24. Velásquez-Meléndez G, Pimenta AM, Kac G. Epidemiologia do sobrepeso e da obesidade e seus fatores determinantes em Belo Horizonte (MG), Brasil: estudo transversal de base populacional. Rev Panam Salud Pública 2004; 16:308-314.

25. Peixoto MRG, Benício MHD’A, Jardim PCBV. The relationship between body mass index and lifestyle in a Brazilian adult population: a cross-sectional survey. Cad Saúde Pública 2007; 23:2694-704.

26. Gigante DP, Barros FC, Post CLA, Olinto MTA. Prevalência de obesidade em adultos e seus fatores de risco. Rev Saúde Pública 1997; 31:236-46.

27. Machado PAN, Sichieri R. Relação cintura-quadril e fatores de dieta em adultos. Rev Saúde Pública 2002; 36:198-204.

28. Silveira CM, Wang Y, Andrade AG, Andrade LH. Heavy episodic drinking in the São Paulo epidemiologic catchment area study in Brazil: gender and sociodemographic correlates. J Stud Alcohol Drugs 2007; 68:18-27.

29. Whitaker RC, Wright JA, Pepe MS, Seidel KD, Dietz WH. Predicting obesity in Young adulthood and parental obesity. N Engl J Med 1997; 337:869-73.

30. Turconi G, Guarcello M, Maccarini L, Zaccardo A, Roggi C. BMI values and other anthropometric and functional measurements as predictors of obesity in a selected group. Eur J Nutr 2006; 45:136-43.

31. Francischi RPP, Pereira LO, Freitas CS, Klopfer M, Vieira P, Lancha Junior AH. Obesidade: atualização sobre sua etiologia, morbidade e tratamento. Rev Nutr 2000; 13:17-28. 
32. Departamento de Análise de Situação em Saúde, Secretaria de Vigilância em Saúde, Ministério da Saúde. Saúde Brasil 2006: uma análise da situação de saúde no Brasil. Brasília: Ministério da Saúde; 2006. (Série G. Estatística e Informação em Saúde).
33. Gus M, Moreira LB, Pimentel M, Gleisener ALM, Moraes FDF. Associação entre diferentes indicadores de obesidade e prevalência de hipertensão arterial. Arq Bras Cardiol 1998; 70:111-4.

Recebido em 05/Mar/2008

Versão final reapresentada em 06/Out/2008

Aprovado em 08/Out/2008 